\title{
Preface: to the special issue: photochemistry and electrochemistry of natural and artificial photosynthesis
}

\author{
Gary F. Moore ${ }^{1}$ Elizabeth Young ${ }^{2}$
}

Published online: 18 February 2022

(c) The Author(s), under exclusive licence to Springer Nature B.V. 2022

Photosynthesis powers our biosphere, enabling plants and other organisms to store the sun's energy as chemical bonds. These bonds constitute the foods we eat and ultimately, on longer geological time scales, the carbon-based fossil fuels our modern societies rely on. Consistent with the latest Intergovernmental Panel on Climate Change report (IPCC 2021), human activities, including the burning of fossil fuels, have pushed the biological process of photosynthesis to its limits and jeopardized multiple Earth systems central to balancing the planet's resource budget.

Rather than exploiting the products of natural photosynthesis, our knowledge of photosynthesis, electrochemistry, and photochemistry could instead be leveraged to develop new technologies with capabilities rivaling those of their biological counterparts. In this special issue of Photosynthesis Research, titled: "Photochemistry and Electrochemistry of Natural and Artificial Photosynthesis," we have gathered a collection of articles from members of the Inter-American Photochemical Society (I-APS) (http://www.i-aps.org/about. asp) that address this outstanding challenge for science and the imagination. Their works highlight the breadth of ongoing research utilizing a range of photochemical and electrochemical techniques to better understand and utilize natural as well as artificial photosynthetic systems.

I-APS was established in 1975 and today has more than 600 members in academia, industry, and government throughout North and South America (Armitage 2013). The mission of I-APS is "to promote and disseminate knowledge,

Elizabeth Young

ery317@lehigh.edu

Gary F. Moore

gary.f.moore@asu.edu

1 School of Molecular Sciences and the Biodesign Institute Center for Applied Structural Discovery (CASD), Arizona State University, Tempe, AZ 85287-1604, USA

2 Department of Chemistry, Lehigh University, Bethlehem, PA 18015, USA and encourage development, of photochemistry and allied subjects throughout the Americas." After years of sponsoring sessions at meetings organized by other organizations, I-APS held its first winter meeting in January 1988 in Clearwater Beach, FL. To this day, I-APS continues to hold an annual Winter Conference, always in a location where photons are plentiful. To celebrate the accomplishments of its members, I-APS has established several prestigious awards to honor the work of distinguished photochemists. I-APS also recognizes that students and post-docs are the future leaders in photochemistry, and supports emerging photochemists. Two awards are currently dedicated to supporting student travel to the I-APS Winter Conferences. I-APS also enthusiastically welcomes membership in the Society by students and strives to facilitate their professional training. This special issue highlights the work of several photochemists who attended one of the recent Winter Conferences. The breadth of research celebrated by I-APS is reflected in the scope of the articles compiled here.

In this issue, Gary Brudvig, Charles Schmuttenmaer, and coworkers review the use of ultrafast terahertz $(\mathrm{THz})$ spectroscopy to provide insights on charge-transfer efficiencies and dynamics in artificial photosynthetic assemblies. This technique provides a non-contact method to measure the ultrafast dynamics and photoconductivity of mobile carriers (electrons and holes) in semiconducting materials, and has proven useful in studying dye-sensitized semiconductor photoelectrodes. Following an introduction to optical-pump THz-probe spectroscopy, a technique that offers both subpicosecond time resolution, the authors discuss three case studies involving use of this technique.

Jillian Dempsey, Elena Jakubikova, and coworkers describe a new Re-complex that undergoes a ligand-tometal charge-transfer (LMCT) transition to form its lowest excited state. Complexes with lowest-energy LMCT excited states are less commonly reported, and therefore more work is necessary to design, measure, and rationalize such systems in order to realize future photochemical applications. 
This newly reported $\left[\operatorname{Re}(\mathrm{dmpe})_{3}\right]^{2+}(\mathrm{dmpe}=$ bis-1,2(dimethylphosphino)ethane) is interrogated both experimentally and computationally using time-dependent density functional theory. Calculations reveal the dominant LMCT transition is highly symmetric and delocalized over all phosphine ligand donors. The computational results provides an understanding for the absence of experimentally observed solvatochromism of $\left[\operatorname{Re}(\mathrm{dmpe})_{3}\right]^{2+}$.

Ksenija Glusac and coworkers review the photophysics of nanographenes. The authors discuss how the size, symmetry, and shape of these materials influence their molecular orbital structures and thus their spectroscopic signatures. Potential applications of nanographenes include light-driven processes ranging from display, lasing, and sensing technologies to photocatalytic water splitting.

Ana Moore, Thomas Moore, and coworkers describe the power of proton-coupled electron transfer (PCET) in moving charge as it relates to developing constructs for artificial photosynthesis. The authors note that within the complex machinery of photosystem II, the redox activity of the tyrosine Z-histidine 190 hydrogen-bonded pair is essential to its functions. This observation motivates their use of charge separation through a PCET mechanism that can compete with reverse charge transfer. Their bioinspired constructs contain a phenol (a model for tyrosine Z) covalently linked to a benzimidazole (a model for histidine) featuring an intramolecular hydrogen bond which closely emulates the one observed in the natural counterpart. They utilize infrared spectroelectrochemistry to identify and measure the oneelectron, one-proton transfer process, and their results provide insights regarding the factors controlling electron and proton motions across relatively long distances while maintaining high redox potentials.

Gary Moore and coworkers report the application of metalloporphyrins, several of which are known to catalyze the hydrogen evolution reaction, as surface coatings in hybrid photoelectrosynthetic assemblies featuring an underlying gallium phosphide $(\mathrm{GaP})$ semiconductor as a light capture and conversion component. In addition to reporting on the synthesis, optical, and electrochemical properties of the homogeneous porphyrin complexes, the authors describe the photoelectrochemistry of the metalloporphyrin-modified $\mathrm{GaP}$ semiconductors. These materials provide opportunities to better understand charge-transfer and carrier-recombination kinetics at semiconductorlcatalystlliquid interfaces.
Valentine Vullev, Daniell Gryko, and coworkers report on the excited-state dynamics of an L-phenylalanine modified corrole. Corroles are aromatic tetrapyrroles with promising properties for use in photonics and biomedical applications (https://doi.org/10.1007/s11120-021-00824-4). In comparison with porphyrins, corroles have a lower molecular symmetry. One result of this lower symmetry is that three pyrrolic protons are distributed among four pyrrolic nitrogens, resulting in distinct tautomers. The tautomerization in the singlet excited state is determined to occur on timescales of 10-100 picoseconds. The authors discuss the potential of picosecond and sub-picosecond charge and energy transfer to occur from high-energy tautomers, thereby granting these reactions an extra $100 \mathrm{meV}$ of thermodynamic driving force.

The research topics and efforts covered in this special issue showcase the potential of using photochemistry and electrochemistry to better understand biological photosynthesis, and to develop human-engineered assemblies inspired by this process. We thank the authors for their excellent contributions to science and to this special edition. Without the time and efforts of reviewers, reporting these works would not have been possible. We therefore offer our sincere thanks to all who provided their time and expertise to offer constructive comments. We are also grateful to Terry M. Bricker (Editor-in-Chief) for his support of I-APS and for the opportunity to showcase exciting work from this community. We imagine a bright future for research development in this area.

\section{References}

Armitage BA (2013) Enlightening the Americas: a history of the InterAmerican Photochemical Society (1975-2013). Photochemistry 41:267-278

http://www.i-aps.org/about.asp

IPCC (2021) Climate Change 2021: the physical science basis. In: Contribution of Working Group I to the Sixth Assessment Report of the Intergovernmental Panel on Climate Change. Cambridge University Press (in press)

Publisher's Note Springer Nature remains neutral with regard to jurisdictional claims in published maps and institutional affiliations. 